\title{
Correction to: Rise and fall of total mesorectal excision with lateral pelvic lymphadenectomy for rectal cancer: an updated systematic review and meta-analysis of 11,366 patients
}

\author{
Gabriele Anania $^{1} \cdot$ Richard Justin Davies $^{2}$ - Alberto Arezzo ${ }^{3}$. Francesco Bagolini ${ }^{1}\left(\mathbb{0} \cdot\right.$ Vito $^{\prime}$ Andrea $^{4}$. \\ Luigina Graziosi $^{5}$. Salomone Di Saverio ${ }^{6}$. Georgi Popivanov ${ }^{7} \cdot$ Isaac Cheruiyot $^{8} \cdot$ Roberto Cirocchi $^{5}$. \\ Annibale Donini ${ }^{5}$
}

Published online: 17 August 2021

(c) Springer-Verlag GmbH Germany, part of Springer Nature 2021

\section{Correction to: International Journal of Colorectal Disease https://doi.org/10.1007/s00384-021-03946-2}

In the original published version of this article, the name of one of the co-author name were incorrectly presented. The author should be presented as Salomone Di Saverio instead of Di Saverio Salomone and cited as Di Saverio S.

The original article has been corrected.

Publisher's Note Springer Nature remains neutral with regard to jurisdictional claims in published maps and institutional affiliations.

The original article can be found online at https://doi.org/10.1007/ s00384-021-03946-2.

Francesco Bagolini

bglfnc@unife.it

Alberto Arezzo

alberto.arezzo@unito.it

Vito D'Andrea

vito.dandrea@uniroma1.it

Georgi Popivanov

gerasimpopivanov@ rocketmail.com

Isaac Cheruiyot

isaacbmn@outlook.com

1 Dipartimento di Scienze Mediche, Università Degli Studi di Ferrara, Ferrara, Italy

2 Cambridge Colorectal Unit - Addenbrooke's Hospital, Cambridge University Hospitals NHS Foundation Trust, Cambridge, UK
3 Department of Surgical Sciences, University of Torino, Corso Dogliotti 14, 10126 Torino, Italy

4 Department of Surgical Sciences, Sapienza University of Rome, Piazzale Aldo Moro 5, 00185 Rome, Italy

5 Department of Surgery and Biomedical Sciences, University of Perugia, 06121 Perugia, Italy

6 Department of General Surgery (S.D.S, University of Insubria, University Hospital of Varese, ASST Sette Laghi, Regione Lombardia, G.I., E.Z., G.C.), Italy

7 Department of Surgery, Ul. "Sv. Georgi Sofiyski” 3, Military Medical Academy, 1606 Sofia, Bulgaria

8 Department of Human Anatomy, University of Nairobi, Nairobi, Kenya 